\title{
Is Cancer Cell Reversion to Normalcy Possible? Un Update
}

ISSN: 2637-773X

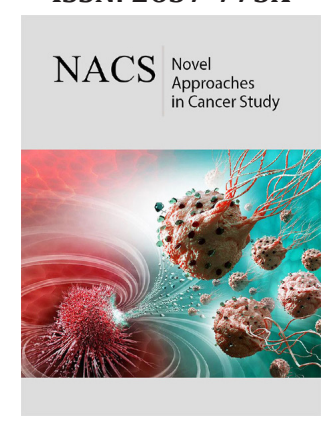

*Corresponding author: Vladimir F Niculescu, Cell biologist, Kirschenweg 1, 86420 Diedorf, Germany

Submission: 㘹: October 09, 2020

Published: 侮 November 02, 2020

Volume 5 - Issue 3

How to cite this article: Vladimir $\mathrm{F}$ Niculescu. Is Cancer Cell Reversion to Normalcy Possible? Un Update. Nov Appro in Can Study. 5(3). NACS.000614. 2020. DOI: 10.31031/NACS.2020.05.000614

Copyright@ Vladimir F Niculescu, This article is distributed under the terms of the Creative Commons Attribution 4.0 International License, which permits unrestricted use and redistribution provided that the original author and source are credited.

\author{
Vladimir F Niculescu* \\ Cell biologist, Germany
}

\section{Introduction}

Molecular biological investigations of the last decade have not been able to definitively determine the source of oncogenic transformation [1]. In recent years, the theory of carcinogenesis by CSCs has become increasingly plausible [2], but where do the CSCs come from? So far, the origin of CSCs has been obscure and controversial [3]. At first, CSCs and normal human stem cells hSCs seem to be strongly related. They have both the capacity for self-renewal and multilineage differentiation. Accordingly, CSCs were thought to originate from normal stem cell mutants, progenitor cell mutants or transiently amplifying cells of unregulated self-renewal ability [4]. Other researchers thought that CSCs originated from adult cells by de-differentiation [5-7] or by de-differentiation of mutated cells that create stem cell-like characteristics. In other words, a fully differentiated cell undergoes mutations or extracellular signals that drive it back to a stem-like state ${ }^{(a)}$. Even today, the origin of CSCs is vague and ambiguous.

In recent years, a less-known type of cancer cell appears to play a pivotal role in cancer development, CSC formation and diversification. It is a polyploid cell, which does not occur in the healthy organism, and resembles giant multinuclear cells of protists (MNGCs, cyst-like structures) [8]. It spreads cancer stem cell-like cells in tumors and tissue. When induced by extrinsic genotoxic agents in established cancer cell cultures, it is referred to as PGCC. Several researchers assume that the PGCC and its numerous daughter cells are strongly related to embryonic cell stages such as the blastula and blastomeres [9-11]. Liu's "life code theory" is an embryonic theory implementing tumorigenesis in the human life cycle. The embryonic theory of carcinogenesis was revived by the cancer stem cell theories [12]. However, it was assumed that CSCs have "fetal" potential and that tumor behavior is expressed by mechanisms of the embryonic development [13].

Over the last decade, more and more researchers resurrected the idea that cancer originates from leftover or restored embryonic cell stages - an idea that goes back to the thinking of Rudolph Virchow [14]. Vinnitski [15] sees the development of a malignant tumor as a desperate asexual self-cloning process in which CSCs develop the ability to mimic the genetic program of germline cells as a potentially endless repetition of asexual life cycles. In Vinnitski's theory, CSCs mimic the behavior of fertilized germline cells of more advanced metazoans which leads to a pseudoblastula producing the tumor [15]. Davies et al. [16] postulate tumors as highly related to Protometazoans (Metazoa 1.0). The authors regard cancer as an atavistic condition that occurs when genetic and epigenetic "malfunction"unlocks an ancient toolkit of preexisting adaptations. They assumed that the development of neoplasms differs significantly from the normal Darwinian theory of evolution. The authors postulate cancer as an atavistic state of multicellular life. The theory of Davies and Linewear was heavily criticized by the proponents of the stochastic theory, which had meanwhile gone out of fashion. A few years later, Han C [17] and his research group at Guangzhou University, China, suggested that cancer is a degenerative evolution state returning to the unicellular ground state, specifically a reversal from multicellularity to unicellularity [18]. They observed the evolution of a human breast-cell-derived-xenograph tumor in mice to understand the evolutionary history of tumorigenesis and found a dominant trend towards the unicellular status of normal embryonic stem cells [19]. 
In 2018 Trigos et al. [20] also suggested that cancer results from a transition from multicellularity to unicellularity through an active constrained process by the disruption of gene regulatory networks, which normally maintain the multicellular state. Jezequel et al. [21] consider tumors to be rather a "pseudomulticellular neotissue ", than a collection of unicellular tumor cells. In their view pseudomulticellularity is a preliminary stage of multicellularity; however, the migrating cells leave solid tumors as unicellular individuals. Concerning multicellularity Bich et al. [22] emphasized that metazoan cells are constrained in multicellular systems to realise and maintain viable identities. When multicellular properties weaken, multicellular regression may occur that is incompatible with the original multicellular cell state [23-25]. Recently, Elizabeth Pennisi reported on hypotheses that consider cancer as a breakdown of multicellularity (doi:10.1126/science. aau5806). On the other hand Libby et al. [26] focuses on the unstable evolutionary transition from the unicellular to the multicellular cell state (MUT) and postulate that so-called molecular "ratches"limit normally regression to unicellularity. However, some multicellular life cycles have required the alternation between unicellular and multicellular life stages. The back-and-forth reversion between early multicellularity and unicellularity brought great evolutionary advantages.

But in the past, cancer was rather seen as a denatured developmental program or as a fault in the developmental chains. It has been suggested that exponential cancer proliferation is decoupled from the terminal cell differentiation, an idea that leads to the hypothesis that cancer cells could potentially re-enter forward differentiation [27]. If cancer would really be a "differentiation disease", it can be assumed that subsequent generations of reverted cancer cells could return to normalcy.

Actually, we have a dispute between two opposing opinions. One assumes that cancer cells would maintain the multicellularity of the cell-of-origin. The high plasticity of cancer cells makes a reversal to normalcy at least conceivable. The other opinion, which regards cancer as a disease of the multicellular cell state leaves little chance for an effective reversal

\section{Cancer "Revertants"}

Two years ago, the issue of tumor and cancer cell regression was discussed in the "News" of the Columbia University, Irving Medical Center (2017) ${ }^{(\mathrm{b})}$. It was about the thoughts of Powers et al. [28], which were published in Nature. Both scientists argue for the idea that cancer cell reversion and the formation of stable revertants could become a suitable tumor reversion therapy. As early as 1968, Pollack et al. [29] described that tumor cells infected with polyomavirus or SV40 achieve a decrease tumor-producing capacity and called them "revertants ". But what are these revertants? In the above discussion, two contradictory definitions are under discussion:

(i) revertants are malignant cells that regain control over their growth or (ii) revertants are cancer cells reverting to normalcy.

The natural incidence of reversion is rare. It is about 1:100,000 or even less, because mutations that cause tumor cells to escape malignancy are rare.

Other research groups are also involved in this discussion. In a 2017 statement by the Universita di Roma, Italian researchers from the SBG Lab ${ }^{(c)}$ believe that cell differentiation is not a state of irreversible commitment, as previously thought, and say „if a somatic cell can be reprogrammed towards its totipotent state, why an abnormal cancer cell should not be reversed becoming so far a "normal" cell? According to these researchers, preliminary studies have shown that special culture media and embryonic-conditioned environments could commit tumor cells to cell differentiation and partial reversion. However, a complete reversion to normal, non-cancerous cells has not been achieved. The so-called "tumor reversion" is not the reversion of malignant transformation.

Over the last three decades, cancer researchers have discovered that certain proteins in tumor cells have different expression (function) when compared to non-cancerous tissue. Some researchers hypothesized that such changes could lead to oncogenic transformation and assumed that the proteins could induce the suppression or reversion of malignancy [30-34]. Reduced tumor-producing capacity was initially observed in fibroblast cell cultures infected with the polyomavirus or SV40. Cells that reduced tumorigenic capacity and arrest growth in G1 were referred to as "cancer revertants". In vivo, spontaneous reversion rates range from 1:100.000 to $1: 1.000 .000$.

\section{Molecular Studies on Tumor Reversion}

In 2002 the Tuynder et al. [33] compared 263 genes that are differentially expressed in "cancer revertants"of breast tumors (263 DE genes). The reversion was induced by the H1 parvovirus, which kills most malignant cells. Cells and clones resistant to the cytopathic effects were isolated and examined for differences in gene expression between

(i) parental malignant cells and their revertants and

(ii) parental cells and stable transfectants transfected with the human SIAH-1 gene after parvovirus selection.

The SIAH-1 gene is a gene that is actively involved in the process of cell death and tumor suppression. The final revertants retain characteristics of the parental cell such as the capacity to undergo multiple passages as well as abnormal chromosomes, but all revertants whether or not they produce the $\mathrm{H} 1$ parvovirus, have reduced their tumorigenicity, similar to the SIAH-1 transfectants. Of the 263 DE genes examined, 32\% were differentially expressed in all tumors. These proteins are either activated or inhibited during reversion. The gene expression profile suggest that the reversion process is not a process of cell cycle arrest or terminal differentiation but rather a process that reprogrammes cancer cells to restore some normal cell functions. The researcher above 
assumes that the reversion occurs primarily at the molecular level and is not the reversion of malignant transformation [34].

Two years later Tuynder et al. [35] identified further revertants from colon, lung, and melanoma tumors, and confirm that persistent infection or expression of $\mathrm{H} 1$ parvovirus is not necessary to suppress malignant phenotypes. The authors believe that "tumor reversion is a biological process, with the capacity to override the oncogenic events ". Accordingly, the revertants have lost almost all their ability to grow and form tumors in mice. A translationally controlled tumor protein gene (TCTP) appears to be the most down-regulated DE gene in this limited "tumor reversion ".

Enane et al. [27] return to the idea that exponential cancer proliferation is decoupled from terminal differentiation, and the appropriate method to stop the malignant proliferation is to reengage terminal differentiation as it occurs before malignant transformation. However, researchers acknowledge that the mechanisms underlying this decoupling are still largely unknown. In their investigations they have tried to identify gene pathways associated with differentiation failures and suggest that the differentiation failures are associated with loss-of-function regarding the master transcription factor (TF) MYC and its cofactors. The unbalanced activity of transcriptional corepressors suggest that these enzymes are targets for tumor cell reversion

\section{"Reversion"by Embryonic Factors is Not Reversion to Normal Cells or Embryonic Phenotypes}

It was shown that embryonic extracts stop proliferation and convert cancer cells into non-invasive cancer cells that have lost the ability to multiply and migrate. In 2019, the group of Mariano Bizzarri showed that embryonic extracts can counteract the migrating invasive phenotype of breast cancer [36]. In the last ten years, a partial reversion of malignant phenotypes was induced by unidentified factors of the embryonic microenvironment $[37,38]$. Soluble factors extracted from human embryonic stem cells and from mouse, chick, fish and amphibian embryos can reverse some features of the malignant phenotype [39], but the revertants are not normal non-cancerous cells. Reversion by embryonic extracts stops cancer cell progression including DNA demethylation, removal of histone repression from tumor suppressor gene promoters and expression of silenced genes [40-43]. According to Proietti et al. [36], embryo-induced reversion is the inhibition of the migratory phenotype adopted by cancerous breast cells undergoing epithelialmesenchymal transition (EMT).

\section{Concluding Remarks}

I agree with the opinion that the reversion of cancer cells to normalcy is as before a "mission impossible ". Embryonic stimuli or pharmacological substances such as antihistaminics, neuroleptics and antidepressants may interrupt the progression of the cancer life cycle or may re-express some silenced genes, but this is not a reversal of oncogenic transformation and return to multicellular normalcy. I believe that cancer cells are definitely bound into the lower level of life of cambrian ancestors capable of multicellularto-unicellular transition (MUT). The life cycle of cancer is a selfsufficient cell system from which cancer cells cannot escape into the more complex multicellular life level of humans and mammalians.

\section{Acknowledgement}

I express my gratitude to Dr. Dennis Thomas from the Cold Spring Harbor Laboratory, USA (native English speaker) for reading the manuscript and improving my English.

\section{References}

(a) https://en.wikipedia.org/wiki/Cancer_stem_cell\#Origin;

(b) https://www.cuimc.columbia.edu/news/can-we-makecancercellsnormal-again;

(c) https://sbglab.org/tumor-reversion.

1. Niculescu VF (2018) Molecular and cell biological considerations in the initiation and development of sporadic non-hereditary solid cancers. J Cancer Genetics and Biomarkers 1(2): 24-40.

2. Fanali C, Lucchetti D, Farina M, Corbi M, Cufino V, etal. (2014) Cancer stem cells in colorectal cancer from pathogenesis to therapy: Controversies and perspectives. World J Gastroenterol 20(4): 923-942.

3. Afify SM, Seno M (2019) Conversion of stem cells to cancer stem cells: Undercurrent of cancer initiation. Cancers 11 (3): 345.

4. Lee CJ, Dosch J, Simeone DM (2008) Pancreatic cancer stem cells. Journal of Clinical Oncology 26(17): 2806-2812.

5. Gangemi R, Paleari L, Orengo AM, Cesario A, Chessa L, et al. (2009) Cancer stem cells: A new paradigm for understanding tumor growth and progression and drug re-sistance. Curr Med Chem 16(14): 1688-1703.

6. Ricci-Vitiani L, Lombardi DG, Pilozzi E, Biffoni M, Todaro M, et al. (2007) Identification and expansion of human colon-cancer-initiating cells. Nature 445(7123): 111-115.

7. O'Brien CA, Pollett A, Gallinger S, Dick JE (20076) A human colon cancer cell capable of initiating tumour growth in immuno-deficient mice. Nature 445(7123): 106-110.

8. Niculescu VF (2019) aCLS cancers: Genomic and epigenetic changes transform the cell of origin of cancer into a tumorigenic pathogen of unicellular organization and lifestyle. Gene 144174.

9. Liu J (2019) The "life code": A theory that unifies the human life cycle and the origin of human tumors. Seminars in Cancer Biology 60: 380397.

10. Niu N, Mercado-Uribe I, Liu J (2017) Dedifferentiation into blastomerelike cancer stem cells via formation of polyploid giant cancer cells. Oncogene 36(34) 4887-4900.

11. Jia L, Zhang S, Ye Y, Li X, Mercado-Uribe I, et al. (2012) Paclitaxel inhibits ovarian tumor growth by inducing epithelial cancer cells to benign fibroblast-like cells. Cancer Lett 326 (2): 176-182.

12. Erenpreisa J, Cragg MS (2008) Life cycle features of tumours cells. Evolutionary Biology from Concept to Application, pp. 61-71.

13. O'Brien-Ball C, Adrian Biddle A (2017) Reprogramming to developmental plasticity in cancer stem cells. Developmental Biology 430(2): 266-274.

14. Shostak S (2011) Evolution of cancer stem cells. In: Stanley Shostak (Ed.), Cancer Stem Cells, The Cutting Edge. Tech, Rijeka, Croatia, p. 557.

15. Vinnitsky V (2014) The development of a malignant tumor is due to a desperate asexual self-cloning process in which cancer stem cells develop the ability to mimic the genetic program of germline cells. Intrinsically Disordered Proteins 2(1): e29997. 
16. Davies PC, Lineweaver CH (2011) Cancer tumors as Metazoa 1.0: Tapping genes of ancient ancestors. Physical biology 8(1): 015001.

17. Han C, Fangqin L, Xionglei H (2014) The degenerative evolution from multicellularity to unicellularity during cancer. Cornell University arXiv, 1408.3236

18. Chen H, Lin F, Xing K, He X (2015) The reverse evolution from multicellularity to unicellularity during carcinogenesis. Nat Commun 6 : 6367.

19. Chen H, Xionglei He X (2016) The convergent cancer evolution toward a single cellular destination. Molecular Biology and Evolution 33(1): 4-12.

20. Trigos AS, Pearson RB, Papenfuss AT, Goode DL (2018) How the evolution of multicellularity set the stage for cancer. Br J Cancer 118: 145-152.

21. Jézéquel $P$, Campone $M$ (2018) Comment on how the evolution of multicellularity set the stage for cancer. British Journal of Cancer 119:133-134.

22. Bich L, Pradeu T, Moreau JF (2019) Understanding mult cellularity: The functional organization of the intercellular space. Front. Physiol 10: 1170 .

23. Sonnenschein C, Soto AM (1999) The society of cells - Cancer and control of cell proliferation. Oxford: Bios Scientifc.

24. Bissell MJ, Radisky D (2001) Putting tumours in context. Nat Rev Cancer 1(1): 46-54.

25. Soto AM, Sonnenschein C (2011) The tissue organization field theory of cancer: A testable replacement for the somatic mutation theory. Bio Essays 33(5): 332-340.

26. Libby E, Conlin PL, KerrB, Ratcliff WC (2016) Stabilizing multicellularity through ratcheting. Philosophical Transactions of the Royal Society of London Series B. Biological Sciences 371(1701): 20150444.

27. Enane FO, Saunthararajah Y, Korc M (2018) Differentiation therapy and the mechanisms that terminate cancer cell proliferation without harming normal cells. Cell Death Dis 9: 912.

28. Poweres S, Polak RE (2016) Inducing stable reversion to achieve cancer control. Nature Review Cancer 16(4): 266-270.

29. Pollack RE, Green H, Todaro GJ (1968) Growth control in cultured cells: selection of sublines with increased sensitivity to contact inhibition and decreased tumor-producing ability. Proc Nat Acad Sci USA 60(1): 126133.

30. Telerman A, Tuynder M, Dupressoir T, Robaye B, Sigaux F, et al. (1993) A model for tumor suppression using H-1 parvovirus. PNAS 90 (18): 8702 8706.

31. Nemani, M, Linares-Cruz G, Bruzzoni-Giovanelli H, Roperch JP, Tuynder M, et al. (1996) Activation of the human homologue of the Drosophila sina gene in apoptosis and tumor suppression. PNAS 93(17): 9039-9042.
32. Roperch JP, Alvaro V, Prieur S, Tuynder M, Nemani M, et al. (1998) Inhibition of presenilin 1 expression is promoted by p53 and p21WAF-1 and results in apoptosis and tumor suppression. Nat Med 4(7): 835-838.

33. Tuynder M, Susini L, Prieur S, Besse S, Fiucci G, et al. (2002) Biological models and genes of tumor reversion: Cellular reprogramming through tpt1/TCTP and SIAH. PNAS 99(23): 14976-14981.

34. Weaver VM, Petersen OW, Wang F, Larabell CA, Briand P, et al. (1997) Reversion of the malignant phenotype of human breast cells in threedimensional culture and in vivo by integrin blocking antibodies. J Cell Biol 137(1): 231-245.

35. Tuynder M, Fiucci G, Prieur S, Lespagnol A, Géant, et al. (2004) Translationally controlled tumor protein is a target of tumor reversion. PNAS 101(43): 15364-15369.

36. Proietti S, Cucina A, Pensotti A, Biava PM, Minini M, et al. (2019) Active fraction from embryo fish extracts induces reversion of the malignant invasive phenotype in breast cancer through down-regulation of TCTP and modulation of E-cadherin/ $\beta$-catenin Pathway. Int J Mol Sci 20(9): 2151.

37. Joel M, Sandberg CJ, Boulland JL, Vik-Mo EO, Langmoen IA, et al. (2013) Inhibition of tumor formation and redirected differentiation of glioblastoma cells in a xenotypic embryonic environment. Dev Dyn 242(9): 1078-1093.

38. Hendrix MJ, Seftor EA, Seftor RE, Kasemeier-Kulesa J, Kulesa PM, et al. (2007) Reprogramming metastatic tumour cells with embryonic microenvironments. Nat Rev Cancer 7(4): 246-255.

39. Biava PM, Canaider S, Facchin F, Bianconi E, Ljungberg L, et al. (2015) Stem cell differentiation stage factors from zebrafish embryo: A novel strategy to modulate the fate of normal and pathological human (stem) cells. Curr Pharm Biotechnol 16(9): 782-792.

40. Hansis C, Barreto G, Maltry N, Niehrs C (2004) Nuclear reprogramming of human somatic cells by xenopusegg extract requires BRG1. Curr Biol 14(16): 1475-1480.

41. Ferranti F, D’Anselmi F, Caruso M, Lei V, Dinicola S, et al. (2013) TCam2 seminoma cells exposed to egg-derived microenvironment modify their shape, adhesive pattern and migratory behaviour: A molecular and morphometric analysis. PLoS ONE 8(10): e76192.

42. D’Anselmi F, Masiello MG, Cucina A, Proietti S, Dinicola S, et al. (2013) Microenvironment promotes tumor cell reprogramming in human breastcancer cell lines. PLoS ONE 8(12): e83770.

43. Allegrucci C, Rushton MD, Dixon JE, Sottile V, Shah M, et al. (2011) Epigenetic reprogramming of breast cancer cells with oocyte extracts. Mol Cancer 10(1): 7. 